\title{
Eye Movement Desensitization and Reprocessing for Posttraumatic Stress Disorder in Bipolar Disorder
}

\author{
Daeyoung $\mathrm{Oh}^{\star}$ and Daeho Kim $\bowtie$ \\ Department of Psychiatry, Hanyang University Medical School, Seoul, Republic of Korea
}

There is evidence that posttraumatic stress disorder (PTSD) is more prevalent in patients with bipolar disorder. According to a review, ${ }^{1}$ the mean prevalence of PTSD in bipolar patients is $16.0 \%$, which is double the lifetime prevalence of PTSD in the general population. ${ }^{2}$ Also bipolar patients with comorbid PTSD exhibit more severe bipolar illness and multiple Axis I disorders, and they disengage more frequently from treatment, suggesting poorer outcome and course of the disorder. ${ }^{3,4}$ Trauma-focused cognitive-behavior therapy and eye movement desensization and reprocessing (EMDR) are considered first-line treatments for PTSD. ${ }^{5}$ Nonetheless, evidence for the efficacy of PTSD treatment in bipolar disorder is lacking. This is an unsatisfactory situation given the fact that antidepressant pharmacotherapy, often suggested as a second-line treatment for PTSD, has limited application for for bipolar patients because of the possibility of manic switch and adverse long-term outcomes. ${ }^{6}$ We report here the successful administration of EMDR to two cases of PTSD in patients with bipolar disorder.

A 25-year-old Korean woman was hospitalized for recurrence of a manic episode that initially began three years earlier. Although her affective symptoms had improved, she was troubled by continuing PTSD symptoms that were initiated by multiple rapes 10 years previously. She was additionally diagnosed with PTSD according to DSM-IV criteria and she scored 52 (mild level of PTSD) on the Clinician-administered PTSD scale (CAPS) ${ }^{7}$; while being maintained on antimanic medi-

Received: June 7, 2013 Revised: August 12, 2013

Accepted: August 21, 2013 Available online: July 21, 2014

$\triangle$ Correspondence: Daeho Kim, MD, PhD

Department of Psychiatry, Hanyang University Medical School, 222 Wangsimni-ro, Seongdong-gu, Seoul 133-791, Republic of Korea

Tel: +82-2-2290-8430, Fax: +82-2-2298-2055

E-mail: dkim9289@hanyang.ac.kr

*Current Affiliation

Department of Psychiatry, CHA Bundang Medical Center, CHA University, Seongnam, Republic of Korea

@ This is an Open Access article distributed under the terms of the Creative Commons Attribution Non-Commercial License (http://creativecommons.org/licenses/bync/3.0) which permits unrestricted non-commercial use, distribution, and reproduction in any medium, provided the original work is properly cited. cations, she was treated with 10 sessions of EMDR starting four weeks after hospitalization and continuing on an outpatient basis, which resulted in complete resolution of PTSD (CAPS score: 7). One year later, she remained in complete remission for PTSD and continued outpatient treatment for bipolar disorder.

A 39-year-old Korean woman with a 20-year history of bipolar disorder was diagnosed with comorbid PTSD, according to DSM-IV criteria, resulting from several traumatic experiences including an accident, sexual assault, and involunatry hospitalization that happened years ago around the time of onset of her bipolar illness. She scored 67 (moderate level of PTSD) on the CAPS and was given nine weekly sessions of EMDR starting one week after admission and continuing through discharge and outpatient treatment. After EMDR, her PTSD symptoms decreased to complete remission (18 on CAPS) and she maintained this therapeutic gain during oneyear of follow-up.

Both patients had PTSD for more than 10 years, and it is unlikely that spontaneous recovery occurred coincidentally with EMDR. Both patients received antimanic and antipsychotic medication concurrently with the EMDR sessions and it is theoretically possible that the recovery was only attributable to pharmacotherapy. However, such second-line medications are not likely to bring about complete remission of chronic PTSD, ${ }^{8}$ and the pharmacotherapy dose remained unchanged over the course of EMDR treatment. Effective (evidence-based) and safe (i.e., unlikely to alter mood or worsen bipolar symptoms, especially during episodes) short-term treatment is needed for PTSD in bipolar disorder.

In the present cases, both patients began EMDR during hospitalization and achived complete remission within 10 sessions. We did not observe any differences in course or response to EMDR compared to other PTSD patients without bipolar disorder. However, for the second patient, who started EMDR while still in a hypomanic state, a difficulty with clinical judgement existed whether processing of memory was complete or she was just optimistic. In this regard, we suggest that EMDR 
be given after affective sympotms become stabilized.

Both patients had mild to moderate PTSD and no other psychiatric conditions. Thus, these cases represent adult-onset simple PTSD rather than complex PTSD, a more severe condition with increased comorbidities caused by repeated childhood interpersonal trauma. Patients with complex PTSD exhibit very limited affect tolerance, dissociative symptoms, features of borderline personality disorders, and suicidal behaviors. In such cases, longer period of stabilization preceding EMDR and further treatment sessions of EMDR may be needed. ${ }^{9}$

In the future, we need to determine whether effective treatment of PTSD symptoms using EMDR can reduce the severity of bipolar disorder and prevent further manic/depressive episodes.

\section{REFERENCES}

1. Otto MW, Perlman CA, Wernicke R, Reese HE, Bauer MS, Pollack MH. Posttraumatic stress disorder in patients with bipolar disorder: a review of prevalence, correlates, and treatment strategies. Bipolar Disord 2004;6:470-479.

2. Kessler RC, Sonnega A, Bromet E, Hughes M, Nelson CB. Posttraumatic stress disorder in the National Comorbidity Survey. Arch Gen Psychiatry 1995;52:1048-1060.

3. Assion HJ, Brune N, Schmidt N, Aubel T, Edel MA, Basilowski M, et al. Trauma exposure and post-traumatic stress disorder in bipolar disorder. Soc Psychiatry Psychiatr Epidemiol 2009;44:1041-1049.

4. Conus P, Cotton S, Schimmelmann BG, Berk M, Daglas R, McGorry $\mathrm{PD}$, et al. Pretreatment and outcome correlates of past sexual and physical trauma in 118 bipolar I disorder patients with a first episode of psychotic mania. Bipolar Disord 2010;12:244-252.

5. Bisson JI, Ehlers A, Matthews R, Pilling S, Richards D, Turner S. Psychological treatments for chronic post-traumatic stress disorder. Systematic review and meta-analysis. Br J Psychiatry 2007;190:97-104.

6. Post RM, Leverich GS, Altshuler LL, Frye MA, Suppes T, McElroy SL, et al. Relationship of prior antidepressant exposure to long-term prospective outcome in bipolar I disorder outpatients. J Clin Psychiatry 2012; 73:924-930.

7. Blake DD, Weathers FW, Nagy LM, Kaloupek DG, Gusman FD, Charney DS, et al. The development of a Clinician-Administered PTSD Scale. J Trauma Stress 1995;8:75-90.

8. Berger W, Mendlowicz MV, Marques-Portella C, Kinrys G, Fontenelle LF, Marmar CR, et al. Pharmacologic alternatives to antidepressants in posttraumatic stress disorder: a systematic review. Prog Neuropsychopharmacol Biol Psychiatry 2009;33:169-180.

9. Korn DL, Leeds AM. Preliminary evidence of efficacy for EMDR Resource Development and Installation in the stablizaiton phase of treatment of complex posttraumatic stress disorder. J Clin Psychol 2002;58: 1465-1487. 